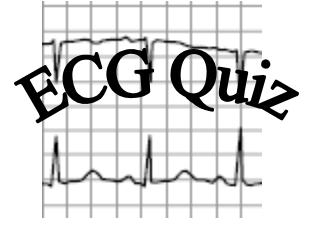

\author{
The Electrocardiogram and \\ Drug-Induced Arrhythmias \\ Bassem Chehab, M.D. \\ Peter Tadros, M.D. \\ University of Kansas Medical Center \\ Cardiovascular Research Institute \\ Cardiovascular Division \\ Kansas City, KS
}

A 72-year-old male with a previous medical history consistent with chronic systolic heart failure presented for frequent episodes of generalized fatigue and shortness of air for the past week. The patient noted that his symptoms started a few days after he began a new anti-fungal medication. The initial echocardiogram is shown below.

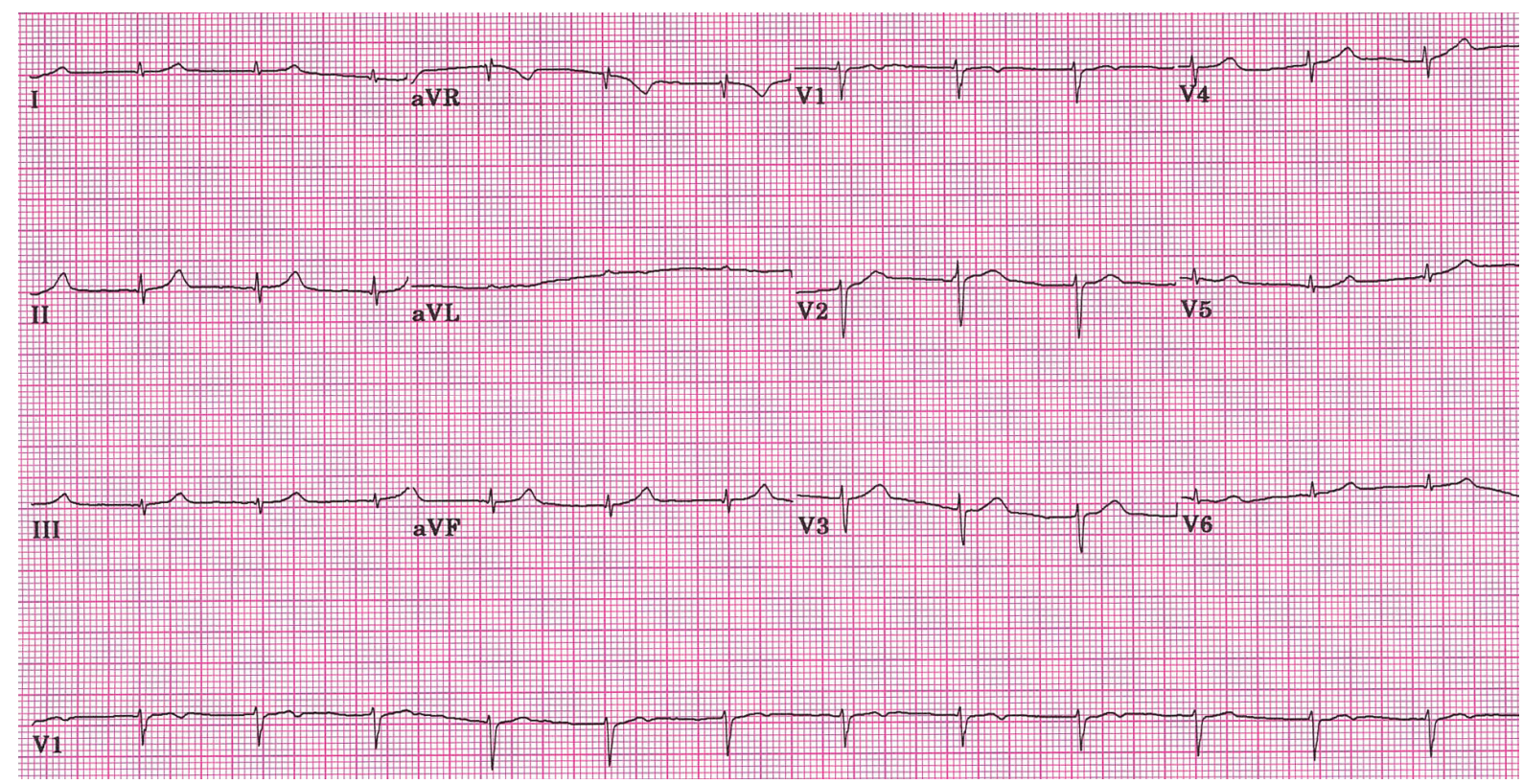

\title{
What is the most likely diagnosis?
}
A) Long QT syndrome
B) Digoxin toxicity
C) Sick sinus syndrome
D) Hypocalcemia 


\section{CORRECT ANSWER: B}

The ECG revealed a narrow QRS complex rhythm at a rate of 78 beats/minute without clearly visualized $\mathrm{P}$ waves, consistent with accelerated junctional rhythm. In the setting of chronic heart failure, this is often caused by digitalis toxicity. Low voltage is evident in the limb leads (QRS amplitude $<5 \mathrm{~mm}$ ) which may be due to pleural effusion, pericardial effusion, or restrictive, infiltrative, or severe ischemic cardiomyopathy. Further questioning revealed this patient was on digoxin therapy and recently started anti-fungal therapy. His diuretic regimen increased which most likely led to his digoxin toxicity.

\section{Discussion}

Digoxin is a complex agent in that its mode of action, inhibition of sodium-potassium adenosine triphosphatase, affects multiple cellular processes, including several critical to cardiac myocyte function. ${ }^{1}$ Digoxin also is extremely toxic, not surprising in view of its apparent role in nature as a toxin evolved by plants to kill mammals. Digoxin has a low therapeutic index and it should be monitored carefully by serum blood levels. The various clinical conditions and drug interactions that can alter digoxin's pharmacokinetics also are reflected in the serum digoxin level. The optimal trough digoxin serum level is 0.5 to $1.0 \mathrm{ng} / \mathrm{mL}$. In patients with heart failure, overt clinical toxicity tends to emerge at serum concentrations greater than $2.0 \mathrm{ng} / \mathrm{mL}$, but substantial overlap in serum levels exists among patients exhibiting symptoms and signs of toxicity and those with no clinical evidence of intoxication.

Disturbances in cardiac impulse formation, conduction, or both are the hallmarks of digitalis toxicity. $^{2}$ Among the common electrocardiographic manifestations are ectopic beats of AV junctional or ventricular origin, first-degree AV block, an excessively slow ventricular rate response to atrial fibrillation, or an accelerated AV junctional pacemaker. These manifestations may require only dosage adjustment and monitoring. Sinus bradycardia, sinoatrial arrest or exit block, and second- or third-degree AV conduction delay often respond to atropine, but temporary ventricular pacing sometimes is necessary and should be available. Neurological or gastrointestinal complaints also can be manifestations of digitalis toxicity. Occasionally, gynecomastia results from digoxin administration, due to similarity with estrogen's structure.

Oral potassium administration often is useful for management of atrial, AV junctional, or ventricular ectopic rhythms, even when the serum potassium is in the normal range, unless highgrade AV block also is present. ${ }^{1,2}$ However, potassium must be monitored carefully to avoid hyperkalemia, especially in patients with renal failure. Magnesium may be useful in patients with atrial fibrillation in an accessory pathway in whom digoxin administration has facilitated a rapid accessory pathway-mediated ventricular response. ${ }^{1}$ Again, careful monitoring is required to avoid hypermagnesemia.

Potentially life-threatening digoxin or digitoxin toxicity can be reversed by antidigoxin immunotherapy. ${ }^{1}$ Purified Fab fragments from digoxin-specific antisera are available at most poison control centers and larger hospitals in North America and Europe. Clinical experience in adults and children has established the effectiveness and safety of antidigoxin Fab in treating life-threatening digoxin toxicity, including cases of massive ingestion with suicidal intent. 


\section{References}

${ }^{1}$ Mann DL. Management of heart failure with reduced ejection fraction. In: Bonow RO, Mann DL, Zipes DP, Libby P (Eds.). Braunwald's Heart Disease: A Textbook of Cardiovascular Medicine. Ninth edition. Philadelphia: WB Saunders, 2011. ISBN 978-1-4377-0398-6.

2 Surawicz B, Knilans T. Chou's Electrocardiography in Clinical Practice: Adult and Pediatric. Sixth edition. Philadelphia: WB Saunders, 2008. ISBN 978-1-4160-3774-3.

Keywords: cardiac arrhythmias, digoxin, toxicity, electrocardiography 